Journal of Advanced Research in Fluid Mechanics and Thermal Sciences

\title{
Turbulent Axisymmetric Non-Isothermal Flow of the Hitec Molten Salt with Temperature Dependent Properties: A Numerical Investigation
}

\author{
Ahmed I. ElShafei ${ }^{1,}$, Amr Guaily ${ }^{1,2,4}$, Mohammed A. Boraey ${ }^{1,3,4}$ \\ Smart Engineering Systems Research Center (SESC), Nile University, Shaikh Zayed City, 12588, Egypt \\ Engineering Mathematics and Physics Department, Faculty of Engineering, Cairo University, Giza, 12613, Egypt \\ 3 Mechanical Power Engineering Department, Zagazig University, Zagazig, 44519, Egypt \\ Mechanical Engineering Program, School of Engineering and Applied Sciences, Nile University, Shaikh Zayed City, 12588, Egypt
}

\section{\begin{tabular}{l} 
ARTICLE INFO ABSTRACT \\
\hline
\end{tabular}}

\section{Article history:}

Received 3 May 2021

Received in revised form 11 July 2021

Accepted 16 July 2021

Available online 19 August 2021

\section{Keywords:}

Temperature-dependent properties; turbulent; axisymmetric; molten salt; Hitec

\begin{abstract}
This study aims to investigate the Hitec molten salt's thermal-hydraulic behavior in a smooth round pipe under broad ranges of surface heat flux and Reynolds number ( $q=$ $\left.10^{4}-10^{5} \mathrm{~W} / \mathrm{m} 2, \operatorname{Re}=10^{4}-10^{5}\right)$. Mesh independent study was performed to ensure the robustness of the model to achieve accurate solutions. Presentation of temperature, pressure and thermophysical properties for multiple cases are presented and discussed. Temperature gradient decreases at high Reynolds number leading to small change in thermo-physical properties. While pressure seems not to be affected by the change in the applied surface heat flux, it increases linearly across the pipe with the increase in Reynolds number. This analysis aims to provide better understanding of the thermal-hydraulic behavior for fluids with temperature dependent properties for a wide range of Re and surface heat flux.
\end{abstract}

\section{Introduction}

Many industries are interested in working fluids undergoing thermal processes and there is continuous research in optimizing the size and cost of heat exchangers and enhancing the rate of heat transfer. Some fluids have pressure and temperature dependent properties with different degrees, the variation of viscosity as a result of temperature change for example is more significant than other properties for most fluids [1]. The most common practice in a lot of studies is to use constant thermophysical properties or only temperature-dependent viscosity fluids to simplify the complexity level of the fluid model with tolerable accuracy since temperature-dependent fluids are more complex to solve than fluids with constant properties [1,2]. However, in the aim of more accurate results and the seek for more optimized solutions with the aid of computational tools, thermophysical temperature-dependent properties of fluids have been recently a topic of engineering interest. The investigations of fluids' variation of properties are conducted either numerically or experimentally. Experimental data are more favorable; however, it is rather expensive

\footnotetext{
* Corresponding author.

E-mail address: AElshafei@nu.edu.eg
}

https://doi.org/10.37934/arfmts.86.2.114 
and time consuming. While numerical solutions have been evolving ever since the advent of computers and numerical techniques and models have been developed for specific problems: wrong solutions will emerge due to the choice of a model incompatible with the studied case. Thus, usually a solid validation is presented in numerical studies to evaluate the accuracy of how the numerical model represents the reality. Several temperature-dependent property fluids studies have been carried out [1-44]. Shah et al., [9], studied the thermal behavior of temperature-dependent fluid in circular cross section as well as Harms et al., [12]. Sehyun et al., [7,13], investigated the variable viscosity's influence on fluids' thermal and hydraulic behavior in rectangular ducts. Berger et al., [13], Andrade et al., [14] and Kumar et al., [15], studied the temperature dependent properties fluids in curved pipes. The latter two mentioned studies reported the water's thermal characteristics in cooling and heating conditions. Watkinson et al., [17], conducted experiments to investigate the forced convection of oils in straight pipes with internal spiral fins. Rainieri et al., [18], experimentally analyzed the thermal performances of temperature-dependent properties Newtonian fluids in axial and helical corrugated tubes with different pitch in the thermal entry region. Garimella et al., [19], showed the dependence of thermal nature of temperature dependent property fluid on flow and geometric variables in laminar, transitional, and turbulent flow regimes in spirally enhanced tubes. Recently more light is shed on new temperature-dependent property material in the fields of Phase Change Material (PCM), Nanofluids and Molten salts, as they are considered potential candidates for concentrated solar power (CSP) plants and thermal energy storage (TES). CSP is an emerging technology that contests other solar technologies such as photovoltaic collectors $[20,21]$. It works on extracting energy from the sun, to harness electric energy, by using a set of mirrors to concentrate the sun rays onto the receivers to heat the heat transfer fluid [22]. Nanofluids have been a topic of interest since they were first discovered by Granqvist et al., [23]. They are considered a prominent thermal energy storage medium as well as PCM [24-27]. Later on, nanofluid preparation techniques were proposed by Li et al., [28]. Choi et al., [29,30], suggested nanofluids as better candidates in heat transfer medium than traditional fluids.

Researchers found a promising potential non pollutant fluid in the energy storage and heat transfer field. Molten salts are favored for their high heat capacity and working temperature, low cost and vapor pressure, and good thermal stability. They are promising candidates to reduce the levelized energy cost of CSP plants. However, there are several drawbacks such as the potential clogging in pipes when the molten salt locally solidifies at night, which will require burning fuel for starting up the plant due to the relatively high melting point. Nevertheless, nitrate-based salts such the commercially known Hitec salt, have several advantages over other molten salt types. Nitrate based salts have lower cost, lower corrosivity and lower melting point than other molten salt types, making it suitable for heat treating plants and thermal energy storage [31]. Multiple experimental work was carried out to investigate the thermophysical properties of multiple kinds of molten salts [32-38]. While other numerical simulations were performed using multiple turbulence models to validate the numerical data with experimental results [39-41]. Finally, an approach by EIShafei et al., [44], to predict the thermal and hydrodynamic behavior of molten salts using Neural networks trained by CFD data is proposed. Since it is very time and money consuming to carry out experimental studies to cover the broad ranges of Reynolds numbers and surface heat flux, numerical simulations are performed to shed more light on the thermal and hydraulic effect on a turbulent temperature dependent property liquid salt flowing in a smooth round pipe using a suitable turbulence model. The present paper is organized as following. Section 2 defines the problem, investigated in the study, by presenting the investigated physical and computational model, the numerical model's governing equations and boundary conditions. 
Mesh independence study and validation with experimental results are conducted in Section 3 and 4 , respectively. In Section 5, the study's results and discussion are given. Finally, a drawn conclusion is given in Section 6 .

\section{Problem Definition}

\subsection{Physical Model}

The modeled physical problem is the flow of molten salt in a circular pipe with uniform surface heat flux. Due to the symmetry of the problem, an axisymmetric model is adopted. The axisymmetric plane of the pipe under investigation is schematically shown in Figure 1. The ternary Hitec molten salt, with the eutectic composition of ( $53 \mathrm{KNO}_{3}-7 \mathrm{NaNO}_{3}-40 \mathrm{NaNO}_{2}$, wt.\%), was used as the flow medium in the simulation analysis. The temperature-dependent thermophysical properties can be described as polynomial piecewise functions of temperature by the below Eq. (1)-(6) (Table 1) [42]. The pipe is $16.6 \mathrm{~mm}$ in diameter and $2000 \mathrm{~mm}$ in length, which was modelled after the DAHAN plant's central receiver's tubes [45]. The pipe is subjected to a uniform heat flux $\left(q=10^{4}-10^{5} \mathrm{~W} / \mathrm{m}^{2}\right)$ on the outer walls and the liquid salt flows at the pipe's inlet with temperature 550k and inlet Reynolds number $\left(\operatorname{Re}=10^{4}-10^{5}\right)$.

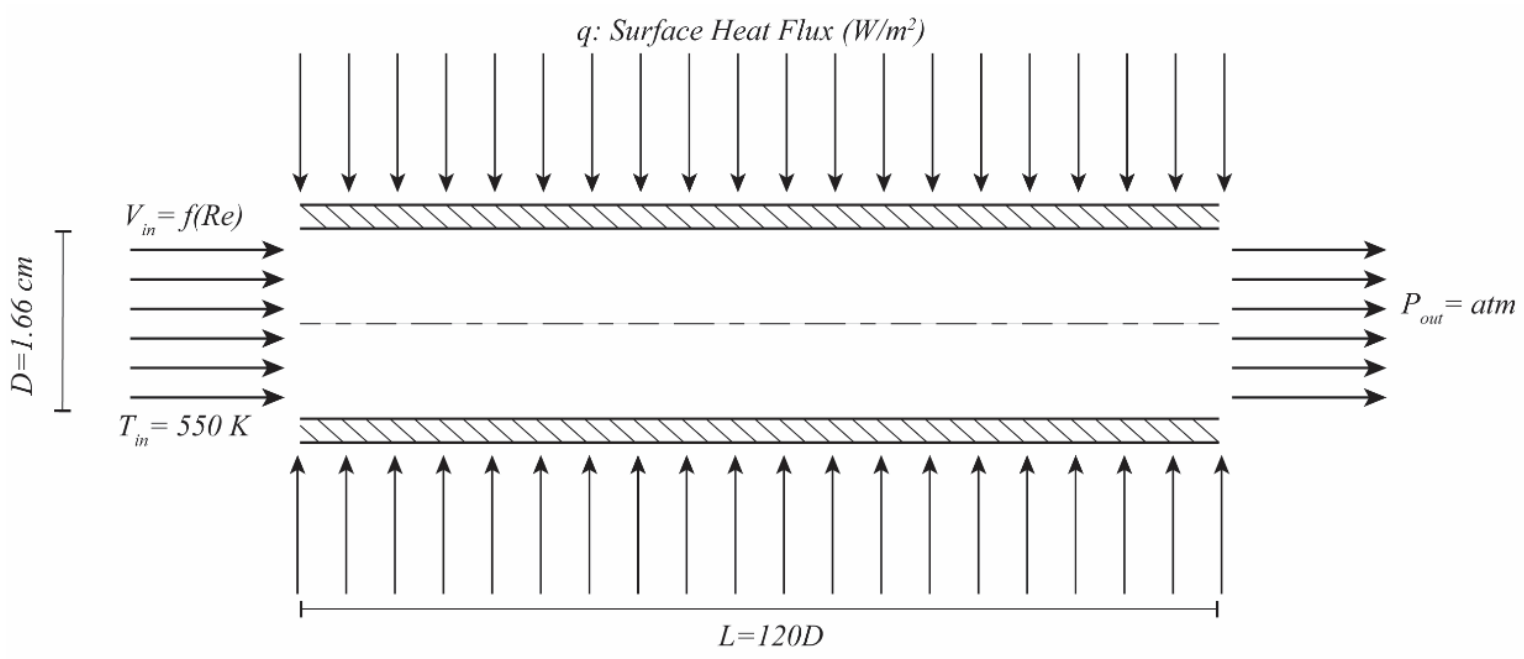

Fig. 1. Schematic description of the presented model

Table 1

Thermophysical properties of Hitec salt

\begin{tabular}{lll}
\hline $\begin{array}{l}\text { Temperature } \\
\text { range }\end{array}$ & Thermophysical property equations & \\
\hline$(450<T<800)$ & $\rho=2280.22-0.636 T$ & $(1)$ \\
$(450<T<800)$ & $C_{p}=1560$ & $(2)$ \\
$(450<T<536)$ & $\lambda=2.2627-0.01176 T+2.551 e-5 T^{2}-1.863 e-8 T^{3}$ & $(3)$ \\
$(536<T<800)$ & $\lambda=0.7663-6.47 e-4 T$ \\
$(450<T<500)$ & $\mu=0.93845-5.4754 e-3 T$ \\
$(500<T<800)$ & $\mu=0.23816-1.2768 e-3 T+2.6275 e-6 T^{2}-2.4331 e-9 T^{3}+8.507 e$ & $(4)$ \\
& $\quad-13 T^{4}$
\end{tabular}

where $T$ represents the salt's temperature, $\rho$ represents the density, $C_{P}$ is the specific heat capacity at constant pressure, $\lambda$ is thermal conductivity and $\mu$ is the dynamic viscosity 


\subsection{Governing Equations}

The basis for the numerical investigation is the solution of the Navier-Stokes, the gravitational effects are neglected, and the energy equation along with the turbulence model equations in addition to the continuity equation.

Mass balance

$\frac{\partial}{\partial x_{i}}\left(\rho u_{i}\right)=0$

\section{Conservation of Momentum}

$\frac{\partial}{\partial x_{i}}\left(\rho u_{i} u_{j}\right)=-\frac{\partial P}{\partial x_{j}}+\frac{\partial}{\partial x_{i}}\left[\left(\mu+\mu_{t}\right)\left(\frac{\partial u_{j}}{\partial x_{i}}+\frac{\partial u_{i}}{\partial x_{j}}-\frac{2}{3} \frac{\partial u_{l}}{\partial x_{l}} \delta_{i j}\right)\right]$

Energy equation

$\frac{\partial}{\partial x_{i}}\left(\rho u_{i} h\right)=\frac{\partial}{\partial x_{i}}\left[C_{p}\left(\frac{\mu}{P r}+\frac{\mu_{t}}{P r_{t}}\right) \frac{\partial T}{\partial x_{i}}\right]$

Turbulence kinetic energy equation

$\frac{\partial\left(\rho k u_{j}\right)}{\partial x_{j}}=\frac{\partial}{\partial x_{j}}\left[\left(\mu+\frac{\mu_{t}}{\sigma_{k}}\right) \frac{\partial k}{\partial x_{j}}\right]+G_{k}-\rho \varepsilon+Y_{M}$

Kinetic energy dissipation rate

$\frac{\partial\left(\rho \varepsilon u_{j}\right)}{\partial x_{j}}=\frac{\partial}{\partial x_{j}}\left[\left(\mu+\frac{\mu_{t}}{\sigma_{\varepsilon}}\right) \frac{\partial \varepsilon}{\partial x_{j}}\right]+\rho C_{1} S \varepsilon-\rho C_{2} \frac{\varepsilon^{2}}{k+\sqrt{v \varepsilon}}$

where $u$ is the salt's velocity, $P$ is the pressure, $x$ represents the coordinates, $h$ is the convective heat transfer coefficient, $P r$ is the salt's Prandtl number, $k$ is turbulent kinetic energy $\& \varepsilon$ is the turbulent dissipation rate.

\subsection{Boundary Conditions}

At the inlet, velocity at the inlet with a uniform profile $u_{i n}$, dependent on the Reynolds number and inlet temperature $T_{i n}=550 \mathrm{~K}$ were used. The inlet turbulent kinetic energy, turbulence intensity and its dissipation rate were estimated using

$k_{\text {in }}=1.5\left(u_{\text {in }} I_{\text {in }}\right)^{2}, I_{\text {in }}=0.16 R e^{-1 / 8}, \varepsilon_{\text {in }}=0.09^{0.75} k_{\text {in }} / 0.07 D$ respectively [46]

No slip condition was imposed on the wall

i. At the surface, uniform wall heat flux boundary $q$ is applied

ii. At the outlet, a fixed atmospheric pressure is employed 


\subsection{Turbulence Modelling}

Previous studies in the literature [39-41] used the standard $k-\varepsilon$ turbulence model with standard wall treatment in predicting the behavior of turbulent molten salt flow in pipes. However, Chen et al., [47], made a comparison between various turbulence models in the prediction of molten salts' heat transfer and friction and concluded that the Realizable $k-\varepsilon$ with Non-Equilibrium Wall Function (NEWF) for near wall treatment is the most accurate model for this type of problems.

The chosen two-equation model was developed by Shih et al., [39] to improve the flow predictions of the standard model for flows that are subjected to rotations, vortices, and strong streamline curvatures. Shih et al., [48] modified the standard model by deriving the dissipation rate's $\varepsilon$ transport equation based on the mean square vorticity fluctuation equation, making the model realizable and consistent with the physics of the turbulent flow [49]. The realizable model's $k-\varepsilon$ transport equations for turbulent kinetic energy and dissipation rate are defined in Eq. (10)-(11). Where the turbulent viscosity is determined by $\mu_{t}=\rho C \mu k^{2} / \varepsilon$. Other terms used in the $k-\varepsilon$ transport equations are defined below.

$G_{k}=\mu_{t} S^{2}$

$C_{1}=\max \left[0.43, \frac{\eta}{\eta+5}\right], \eta=S \frac{k}{\varepsilon}, S=\sqrt{2 S_{i j} S_{i j}}$

$C_{1 \varepsilon}=1.44, C_{2}=1.9, \sigma_{k}=1, \sigma_{\varepsilon}=1.2$

where $G_{k}$ is a term representing the turbulence kinetic energy's generation due to mean velocity gradient and $S$ represents the mean strain rate tensor modulus [50].

\subsection{Computational Model}

A 2D axisymmetric domain was used due to the axisymmetric nature of the physical model's geometry, flow, and heat flux. The fluid and solid regions were meshed using structured quadrilateral elements as shown in Figure 2.

The numerical simulation is performed using the Ansys Fluent commercial code to solve the flow's governing Eq. (7)-(12) over the presented grid in Figure 2. Segregated SIMPLE algorithm [41] was used for the pressure-velocity coupling and second order upwind schemes were applied for the discretization of the momentum, energy, and turbulence model equation. Residual values were monitored and a value of $10^{-6}$ is set as a convergence criterion.

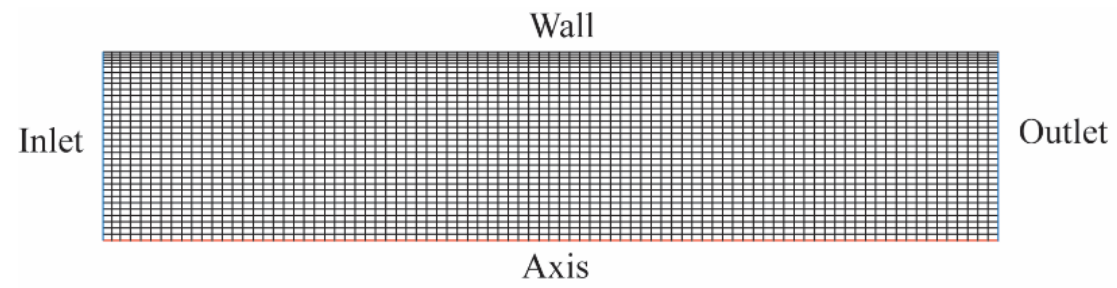

Fig. 2. Computational grid model 


\section{Mesh Independence Study}

Grid independence analysis was carried out for the case which is subjected to maximum temperature gradient with $\mathrm{Re}_{\mathrm{in}}=10 \mathrm{k}, \mathrm{q}=100 \mathrm{~kW} / \mathrm{m}^{2}$ at five different grid sizes $22 \mathrm{k}, 54 \mathrm{k}, 88 \mathrm{k}, 150 \mathrm{k}$ $\& 216 \mathrm{k}$ elements. The temperature at the centerline and the wall was monitored at these different grids, as shown in Figure 3(a). Moreover, the centerline velocity was also monitored as shown in Figure 3(b). It can be noticed in Figure 3 that the $22 \mathrm{k}$ element grid is not suitable since the velocity profile is significantly apart from the rest of the grids. While the $54 \mathrm{k}$ element grid has a small percentage of error with the higher element grids. It can be observed that the $88 \mathrm{k}$ element grid has a very little to no deviation from the grids of higher elements, for both temperature and velocity profiles. Thus, to compromise between the simulation time usage and accurate results the $88 \mathrm{k}$ element grid was used to perform the rest of the simulation cases.

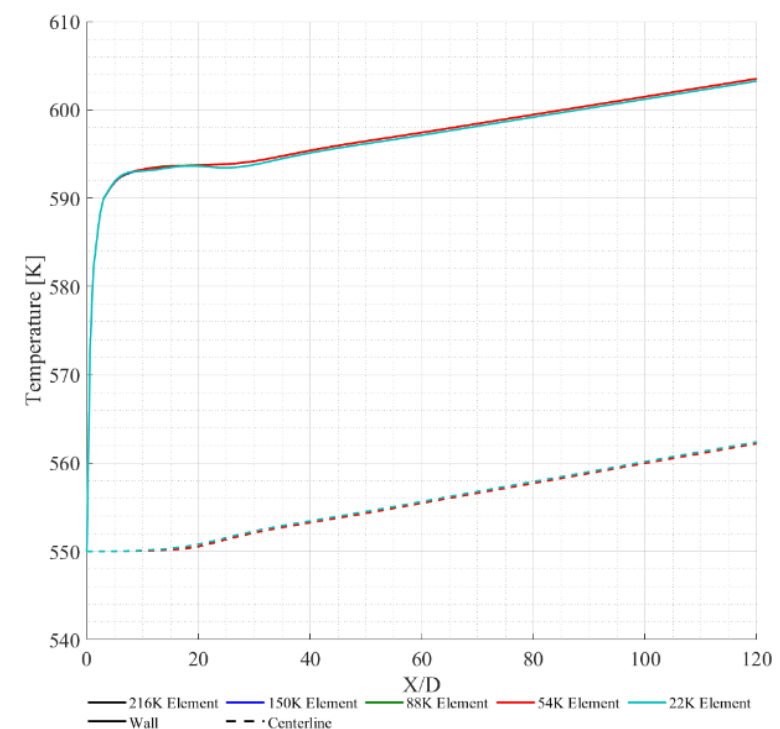

(a)

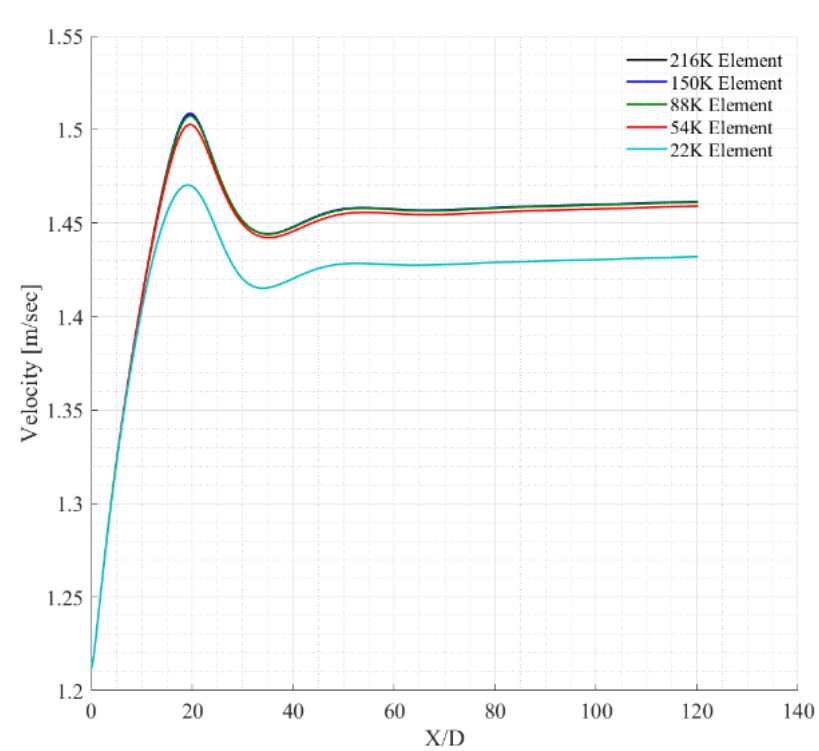

(b)

Fig. 3. Grid independence study for wall temperature for 5 different grid (a) Temperature comparison (b) Velocity comparison

\section{Numerical Model Validation}

Based on the chosen number of elements from the mesh independence test, the numerical model was validated using the experimental and simulation Nusselt number results generated by Qiu et al., [45]. The validation was done under the same geometrical and boundary conditions, under $100 \mathrm{~kW} / \mathrm{m}^{2}$ uniform heat flux and 10,000-20,000 Reynolds number. Figure 4 shows a good agreement between the simulation's results and Qiu's data. The shift from the experimental data can be attributed to heat losses, the non-uniformity of the heat flux and the fact that the properties of molten salt used for the experiment can vary slightly from the molten salt used in the numerical simulation. 


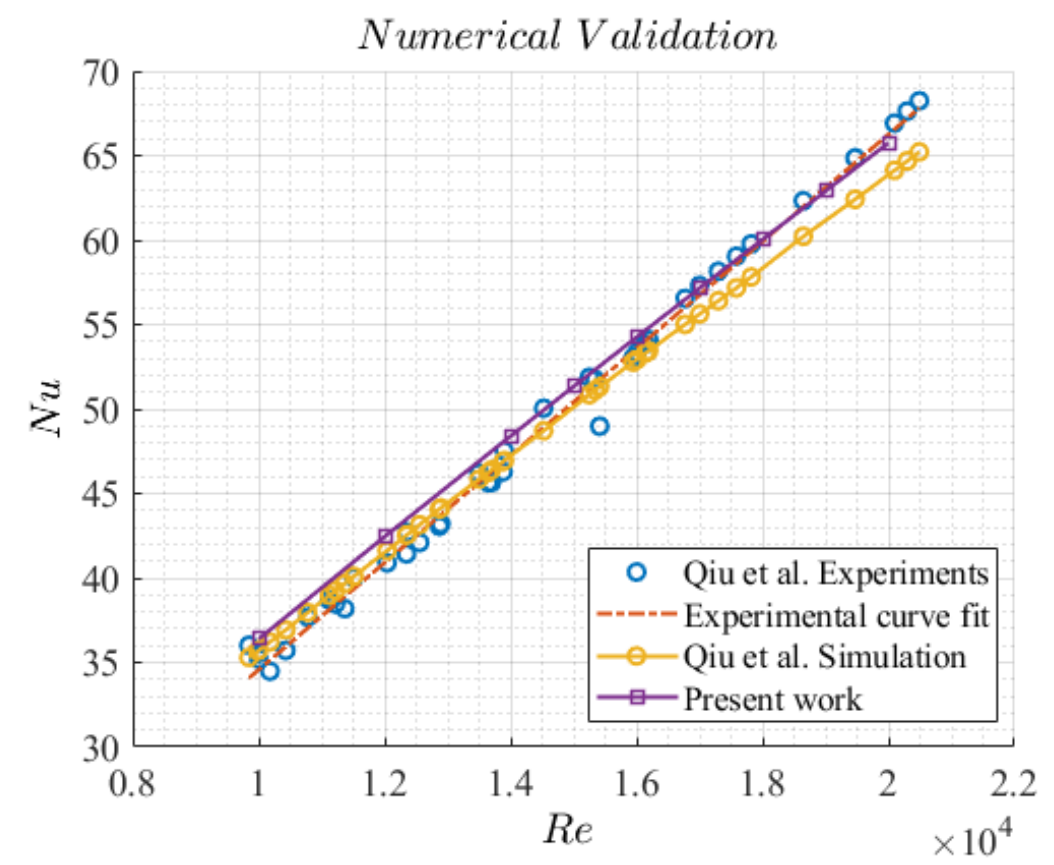

Fig. 4. Average Nusselt number validation for the numerical model

\section{Results and Discussion}

In this section, results of temperature, pressure and density between multiple cases are presented and discussed.

\subsection{Comparison for Constant Heat flux for Variable Reynolds Numbers}

Figure 5 illustrates the change of density due to temperature at $q=100 \mathrm{~kW} / \mathrm{m}^{2}$ and different Reynolds number, it can be observed that density gradient across the pipe's centerline is high at low Reynolds number and decreases as the Reynolds number increase. Figure 6 displays the molten salt's velocity contours. Figure 7 illustrates the local change of density due to the variation of temperature. Figure 8 shows the variation in the molten salt's dynamic viscosity due to the variation of temperature, defined by the thermophysical temperature dependent properties presented in Table 1. This can be explained by Figure 9-11 that displays the temperature fields across the pipe. In the temperature contours in Figure 9-11, the temperature gradient can be seen to be more remarkable at the wall near the pipe walls at lower Reynolds number for the same heat flux values and that average radial temperature gradient decreases with the increase in Reynolds number. It is noticed in Figure 12, that the pressure along the pipe increases with the increase of Reynolds number. Moreover, the pressure linearly decreases linearly with the axial distance. Exhibited in Figure 13, the rate of change of axial temperature at the centerline decreases with the increase of Reynolds number which was noticed in the temperature contour profiles. 


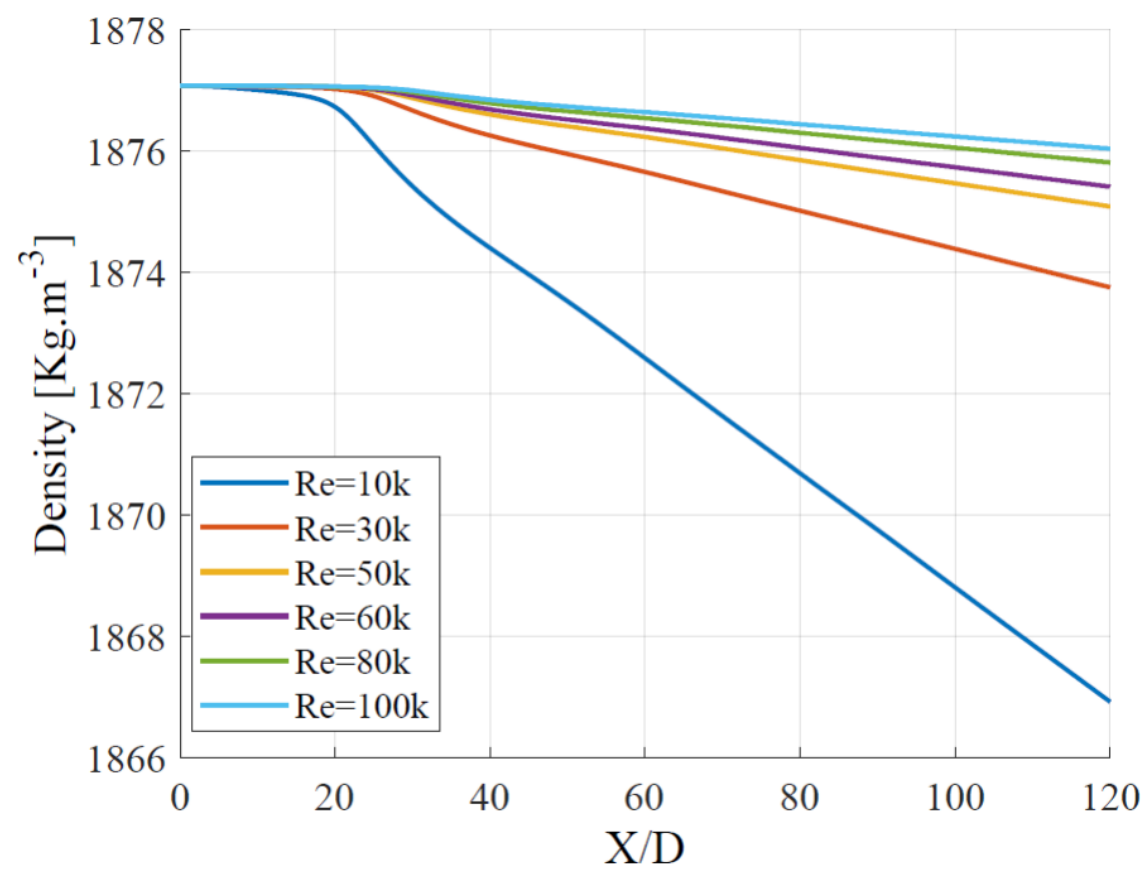

Fig. 5. Density across the pipe's Centerline at heat flux $=100 \mathrm{~kW} / \mathrm{m}^{2}$

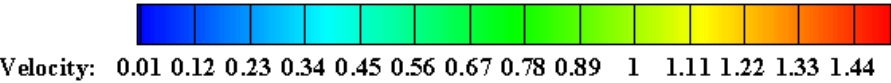

Fig. 6. Velocity across the pipe's Centerline at heat flux $=100 \mathrm{~kW} / \mathrm{m}^{2}$

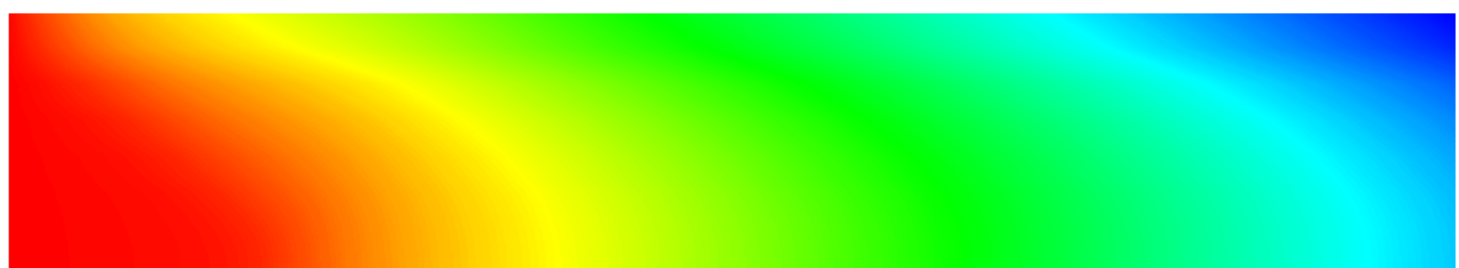

Density: $1865.95 \quad 1867.55 \quad 1869.15 \quad 1870.75 \quad 1872.35 \quad 1873.95 \quad 1875.55$

Fig. 7. Density contour for $\operatorname{Re}=10 \mathrm{k} \& \mathrm{q}=100 \mathrm{~kW} / \mathrm{m}^{2}$

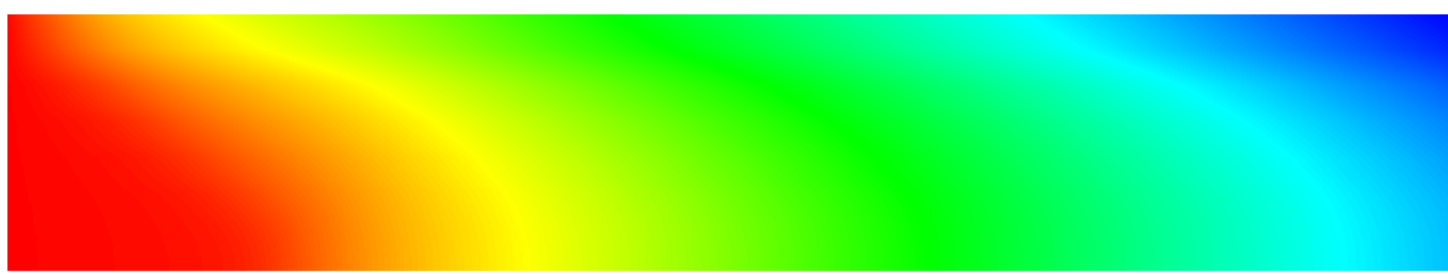

$\begin{array}{lllllll}\text { Viscosity: } & 0.003378 & 0.003446 & 0.003514 & 0.003582 & 0.00365 & 0.003718\end{array}$

Fig. 8. Viscosity contour for $\operatorname{Re}=10 \mathrm{k} \& \mathrm{q}=100 \mathrm{~kW} / \mathrm{m}^{2}$ 


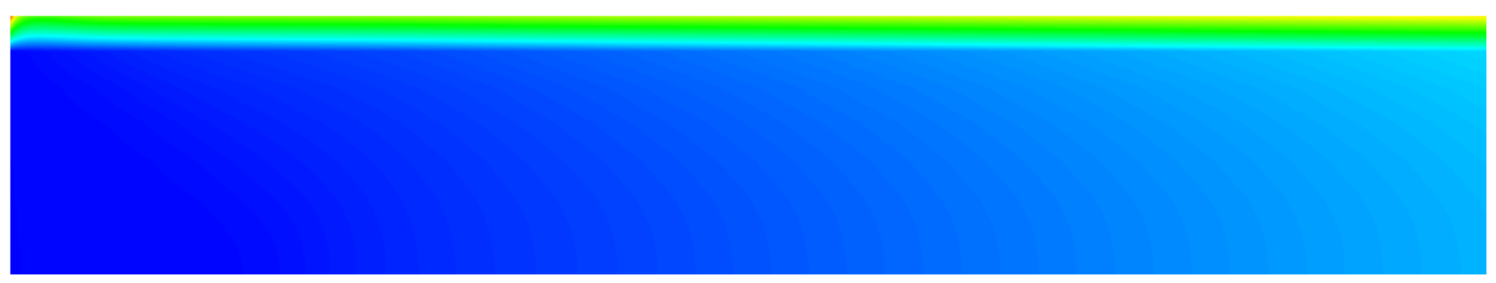

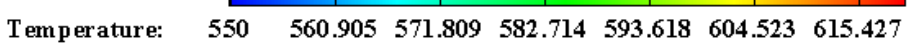

Fig. 9. Temperature contour for $\operatorname{Re}=10 \mathrm{k} \& \mathrm{q}=100 \mathrm{~kW} / \mathrm{m}^{2}$
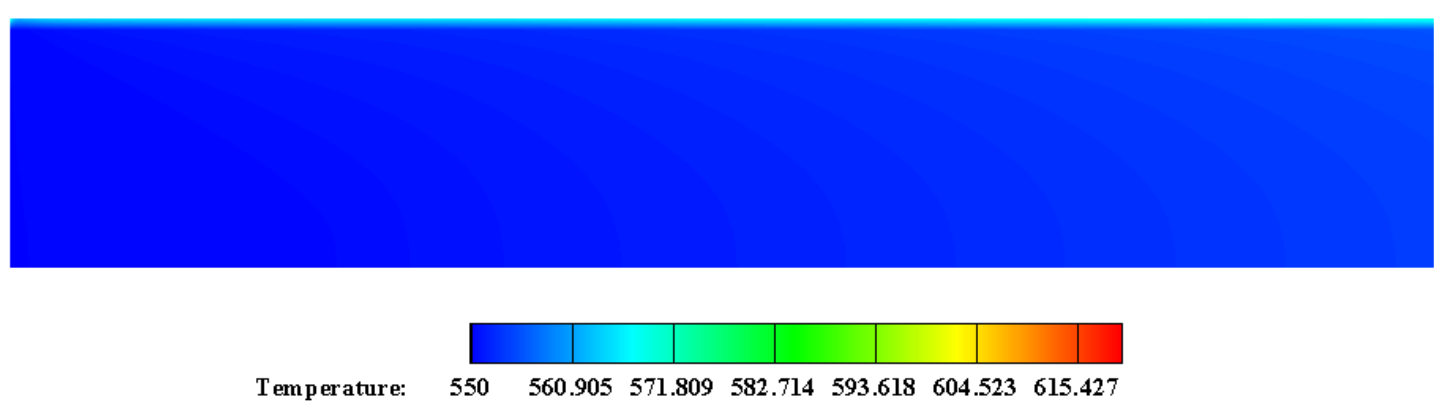

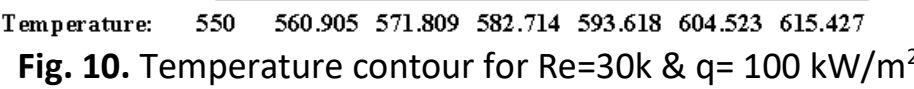

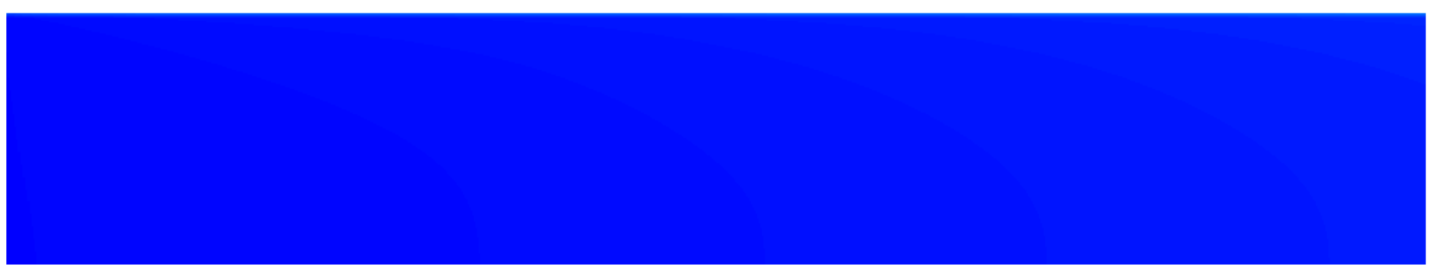

$\begin{array}{llllllll}\text { Temperature: } & \mathbf{5 5 0} & \mathbf{5 6 0 . 9 0 5} & \mathbf{5 7 1 . 8 0 9} & \mathbf{5 8 2 . 7 1 4} & \mathbf{5 9 3 . 6 1 8} & \mathbf{6 0 4 . 5 2 3} & \mathbf{6 1 5 . 4 2 7}\end{array}$

Fig. 11. Temperature contour for $\operatorname{Re}=80 \mathrm{k} \& \mathrm{q}=100 \mathrm{~kW} / \mathrm{m}^{2}$

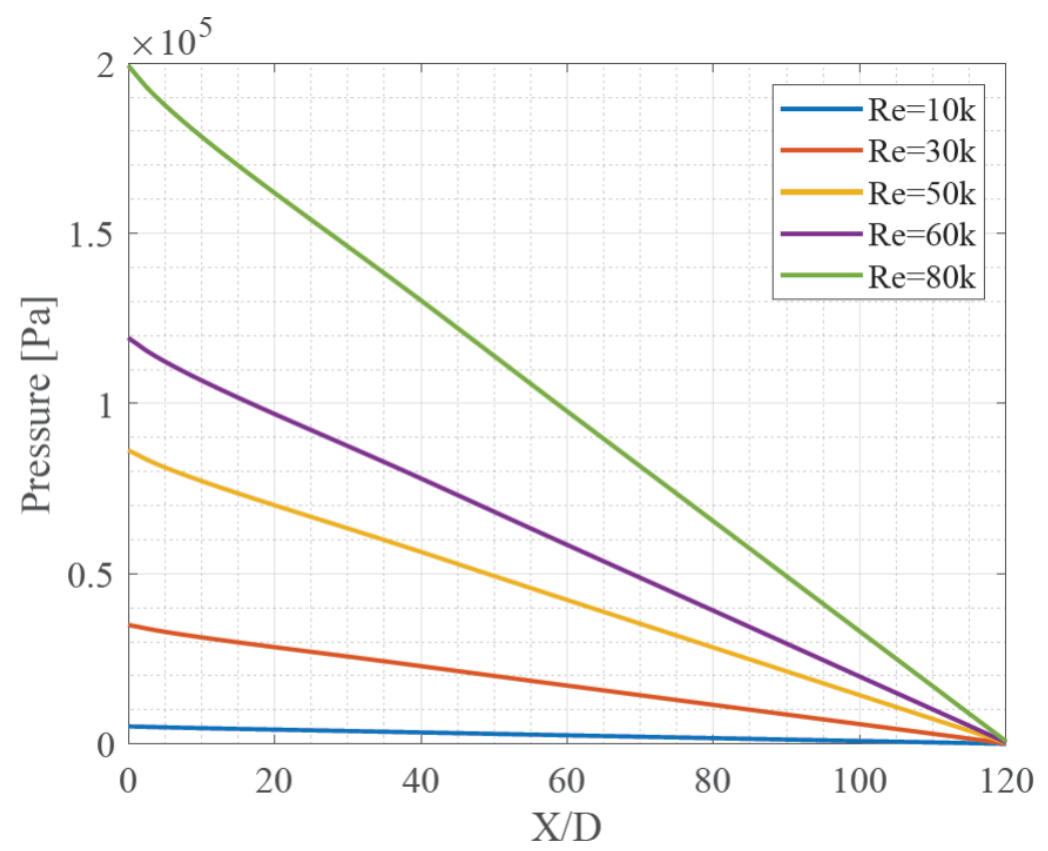

Fig. 12. Pressure across the pipe at heat flux $=100 \mathrm{~kW} / \mathrm{m}^{2}$ 


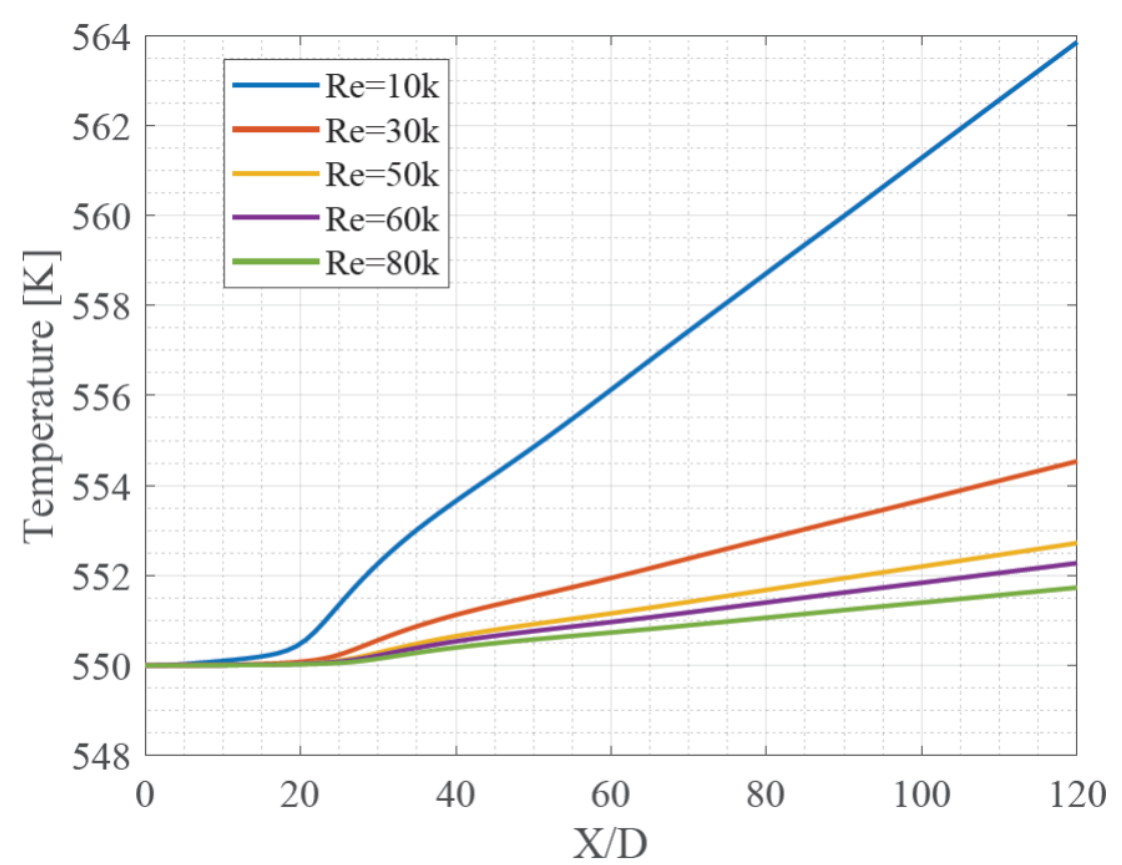

Fig. 13. Temperature across the pipe's Centerline at heat flux $=100$ $\mathrm{kW} / \mathrm{m}^{2}$

\subsection{Comparison for Constant Reynolds Number for Variable Heat Flux}

Figure 14 illustrates the change of density due to temperature at $\mathrm{Re}=50 \mathrm{k}$ and different surface heat flux, it can be observed that density gradient along the pipe varies due to different applied heat fluxes that resulted in different temperature distribution across the pipe. It is also noticed in Figure 15, that the pressure along the pipe does not change with the change in heat flux. Exhibited in Figure 16, the gradient of the axial temperature at the centerline decreases with the decrease of surface heat flux which was noticed in the temperature contour profiles. The temperature at the centerline does not change for the first portion of the pipe which was reflected on the molten salt's temperature dependent density in Figure 14.

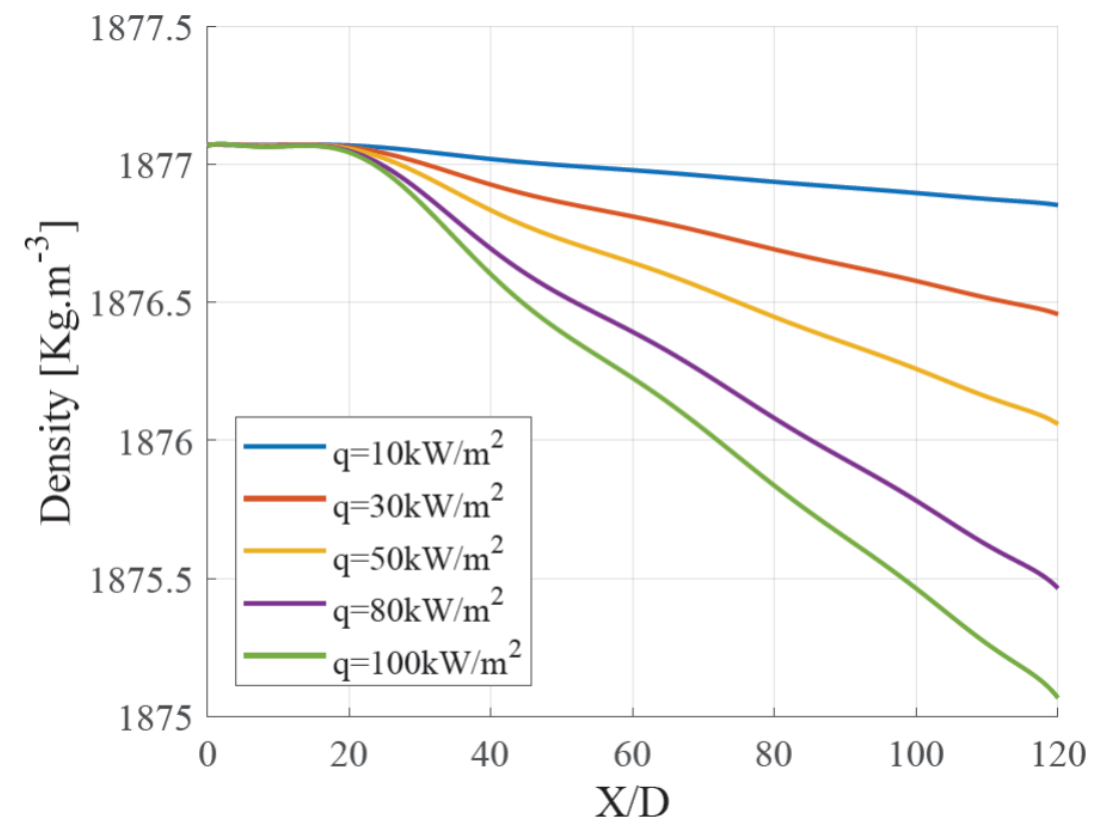

Fig. 14. Density across the pipe's Centerline at $\mathrm{Re}=50 \mathrm{k}$ 
The first portion of the temperature does not change because the thermal boundary layer has not reached the pipe's center. Therefore, the flow is considered not thermally developed yet. Thermal entry length is proportional to both the Reynolds number and the fluid's Prandtl number. It can be seen in Figure 13 that it takes the fluid at higher Reynolds number more axial distance to reach the thermal developed state, which explains why the distance of the constant temperature at the pipe's inlet region increases as the Reynolds number increase. However, in Figure 16 the change in the thermal development length is very un-noticeable.

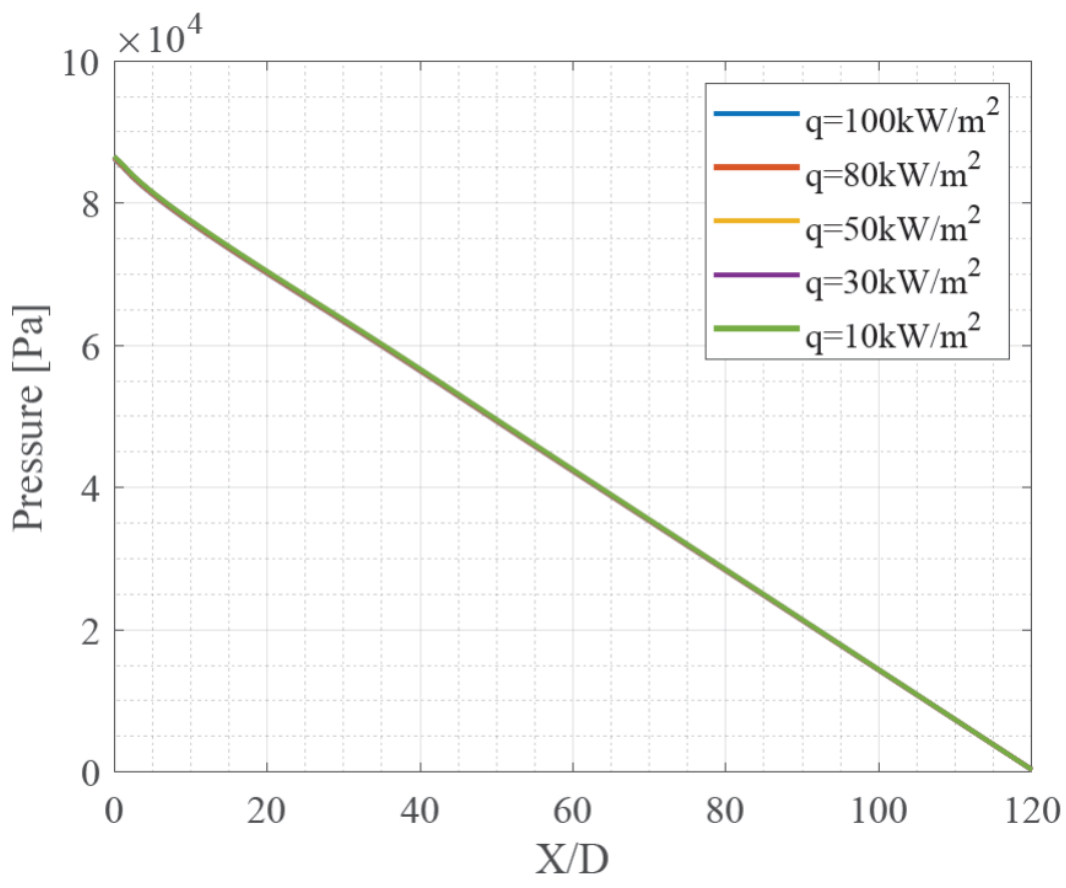

Fig. 15. Pressure across the pipe at $R e=50 k$

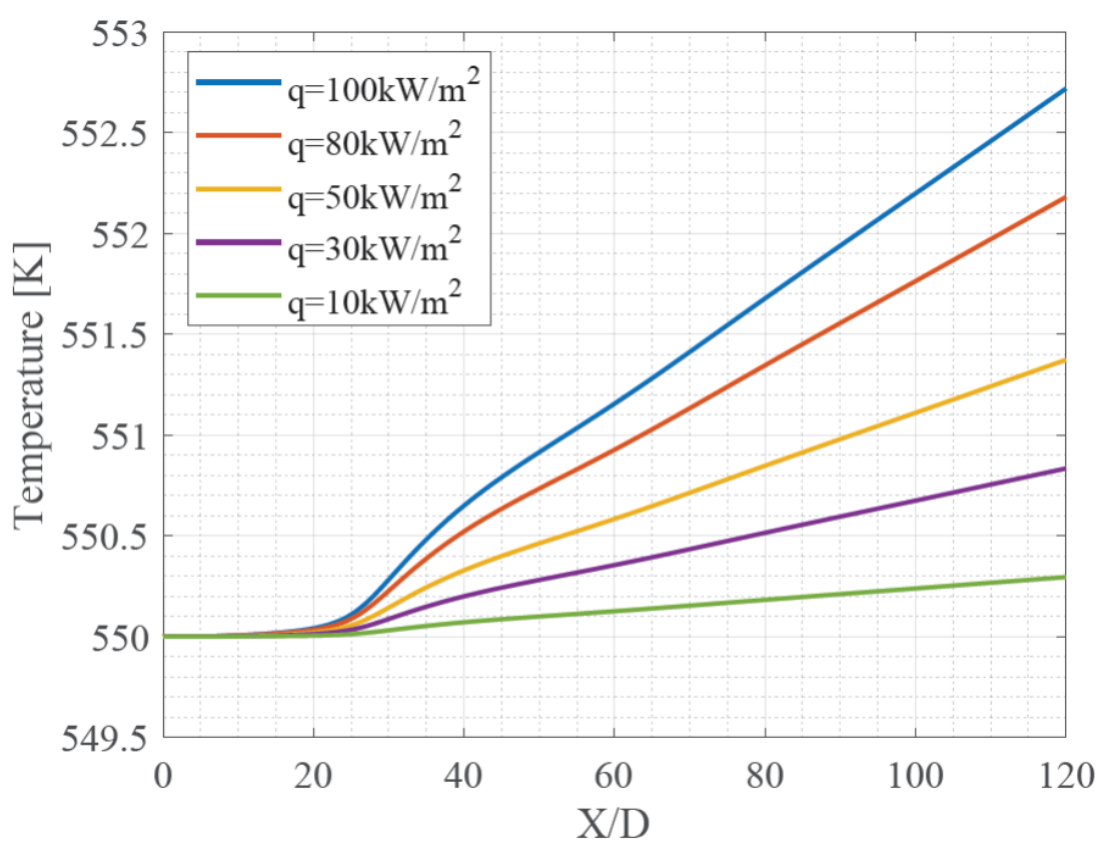

Fig. 16. Temperature across the pipe's Centerline at $\mathrm{Re}=50 \mathrm{k}$ 


\section{Conclusion}

The thermal and hydrodynamic behavior of the temperature dependent properties fluid, Hitec molten salt, is analyzed numerically, after obtaining a grid independent solution, under the effect of wide ranges of inlet Reynolds number and surface heat flux using the realizable $k-\varepsilon$ turbulence model with NEWF near wall treatment. The obtained conclusions of the study are

i. Temperature gradient decreases with the increase in Reynolds number

ii. As a result of decrease in temperature gradient, gradient of thermophysical properties like density also decreases

iii. The pressure along the pipe increases almost linearly across the pipe with the increase of Reynolds number

iv. Applied surface heat flux has no effect on the pressure gradient across the pipe

\section{References}

[1] Wang, Cuihua, Shengju Liu, Jianhua Wu, and Zhang Li. "Effects of temperature-dependent viscosity on fluid flow and heat transfer in a helical rectangular duct with a finite pitch." Brazilian Journal of Chemical Engineering 31 (2014): 787-797. https://doi.org/10.1590/0104-6632.20140313s00002676

[2] Nicoll, William Burns, and W. M. Kays. The Influence of Temperature Dependent Properties on Gas Flow Heat Transfer in Circular Tubes. STANFORD UNIV CA, 1959.

[3] Wichterle, Kamil. "Heat transfer in temperature-dependent flow." The Chemical Engineering Journal 26, no. 3 (1983): 217-222. https://doi.org/10.1016/0300-9467(83)80016-1

[4] Xie, C., and J. P. Hartnett. "Influence of variable viscosity of mineral oil on laminar heat transfer in a 2: 1 rectangular duct." International journal of heat and mass transfer 35, no. 3 (1992): 641-648. https://doi.org/10.1016/00179310(92)90123-A

[5] Sieder, E. Nn, and Go E. Tate. "Heat transfer and pressure drop of liquids in tubes." Industrial \& Engineering Chemistry 28, no. 12 (1936): 1429-1435. https://doi.org/10.1021/ie50324a027

[6] Deissler, Robert G. Analytical investigation of fully developed laminar flow in tubes with heat transfer with fluid properties variable along the radius. NATIONAL AERONAUTICS AND SPACE ADMINISTRATION WASHINGTON DC, 1951.

[7] Sehyun, Shin, Young I. Cho, William K. Gringrich, and Wei Shyy. "Numerical study of laminar heat transfer with temperature dependent fluid viscosity in a 2: 1 rectangular duct." International journal of heat and mass transfer 36, no. 18 (1993): 4365-4373. https://doi.org/10.1016/0017-9310(93)90121-L

[8] Zonta, Francesco, and Alfredo Soldati. "Effect of temperature dependent fluid properties on heat transfer in turbulent mixed convection." Journal of heat transfer 136, no. 2 (2014). https://doi.org/10.1115/1.4025135

[9] Shah, Ramesh K., and Alexander Louis London. Laminar flow forced convection in ducts: a source book for compact heat exchanger analytical data. Academic press, 2014.

[10] Herwig, H. "The effect of variable properties on momentum and heat transfer in a tube with constant heat flux across the wall." International journal of heat and mass transfer 28, no. 2 (1985): 423-431. https://doi.org/10.1016/0017-9310(85)90075-4

[11] Herwig, H., M. Voigt, and F-J. Bauhaus. "The effect of variable properties on momentum and heat transfer in a tube with constant wall temperature." International Journal of Heat and Mass Transfer 32, no. 10 (1989): 1907-1915. https://doi.org/10.1016/0017-9310(89)90160-9

[12] Harms, T. M., M. A. Jog, and R. M. Manglik. "Effects of temperature-dependent viscosity variations and boundary conditions on fully developed laminar forced convection in a semicircular duct." (1998): 600-605. https://doi.org/10.1115/1.2824317

[13] Berger, S. A., L. Talbot, and L. S. Yao. "Flow in curved pipes." Annual review of fluid mechanics 15, no. 1 (1983): 461512. https://doi.org/10.1146/annurev.fl.15.010183.002333

[14] Andrade, Claudia R., and Edson L. Zaparoli. "Effects of temperature-dependent viscosity on fully developed laminar forced convection in a curved duct." International communications in heat and mass transfer 28, no. 2 (2001): 211220. https://doi.org/10.1016/50735-1933(01)00228-7

[15] Kumar, Vimal, Pooja Gupta, and K. D. P. Nigam. "Fluid flow and heat transfer in curved tubes with temperaturedependent properties." Industrial \& engineering chemistry research 46, no. 10 (2007): 3226-3236. https://doi.org/10.1021/ie0608399 
[16] Wang, Cuihua, Shengju Liu, Jianhua Wu, and Zhang Li. "Effects of temperature-dependent viscosity on fluid flow and heat transfer in a helical rectangular duct with a finite pitch." Brazilian Journal of Chemical Engineering 31 (2014): 787-797. https://doi.org/10.1590/0104-6632.20140313s00002676

[17] Watkinson AP, Miletti DI, And Kubanek GR. "Heat transfer and pressure drop of internally finned tubes in turbulent air flow." (1975).

[18] Rainieri, S., and G. Pagliarini. "Convective heat transfer to temperature dependent property fluids in the entry region of corrugated tubes." International Journal of Heat and Mass Transfer 45, no. 22 (2002): 4525-4536. https://doi.org/10.1016/S0017-9310(02)00156-4

[19] Garimella, Srinivas, and Richard N. Christensen. "Performance evaluation of spirally fluted annuli: geometry and flow regime effects." Heat transfer engineering 18, no. $1 \quad$ (1997): $34-46$. https://doi.org/10.1080/01457639708939888

[20] Abdullah, Amira Lateef, Suhaimi Misha, Noreffendy Tamaldin, Mohd Afzanizam Mohd Rosli, and Fadhil Abdulameer Sachit. "Numerical analysis of solar hybrid photovoltaic thermal air collector simulation by ANSYS." CFD Letters 11 , no. 2 (2019): 1-11.

[21] Memon, Saim, Takao Katsura, Ali Radwan, Shanwen Zhang, Ahmed A. Serageldin, Essam M. Abo-Zahhad, Sergeev Sergey et al. "Modern eminence and concise critique of solar thermal energy and vacuum insulation technologies for sustainable low-carbon infrastructure." International Journal of Solar Thermal Vacuum Engineering 1, no. 1 (2020): 52-71. https://doi.org/10.37934/stve.1.1.5271

[22] Shatnawi, Hashem, Chin Wai Lim, Firas Basim Ismail, and Abdulrahman Aldossary. "Numerical Study of Heat Transfer Enhancement in A Solar Tower Power Receiver, Through the Introduction of Internal Fins." Journal of Advanced Research in Fluid Mechanics and Thermal Sciences 74, no. 1 (2020): 98-118. https://doi.org/10.37934/arfmts.74.1.98118

[23] Granqvist, C. G., R. A. Buhrman, J. Wyns, and A. J. Sievers. "Far-infrared absorption in ultrafine Al particles." Physical Review Letters 37, no. 10 (1976): 625. https://doi.org/10.1103/PhysRevLett.37.625

[24] Sudharsan, Natteri Mangadu. "Melting rate analysis for optimization of fin configuration in PCM based thermal energy storage system." Journal of Advanced Research in Fluid Mechanics and Thermal Sciences 78, no. 2 (2021): 34-46. https://doi.org/10.37934/arfmts.78.2.3446

[25] Jamil, Nurfatihah, Jesbains Kaur, A. K. Pandey, Syed Shahabuddin, Samir Hassani, Rahman Saidur, Roshafima Rasit Ali, Nor Azwadi Che Sidik, and Mohd Naim. "A review on nano enhanced phase change materials: an enhancement in thermal properties and specific heat capacity." Journal of Advanced Research in Fluid Mechanics and Thermal Sciences 57, no. 1 (2019): 110-120.

[26] Kee, Chow Hoong, Nor Azwadi Che Sidik, Siti Nurul Akmal Yusof, M'hamed Beriache, and Ahmad Tajuddin Mohamad. "Performance enhancement of cold thermal energy storage system using nanofluid phase change materials." Journal of Advanced Research in Applied Mechanics 62, no. 1 (2019): 16-32.

[27] Kean, Tung Hao, and Nor Azwadi Che Sidik. "Thermal performance analysis of nanoparticles enhanced phase change material (NEPCM) in cold thermal energy storage (CTES)." CFD Letters 11, no. 4 (2019): 79-91.

[28] Li, Yanjiao, Simon Tung, Eric Schneider, and Shengqi Xi. "A review on development of nanofluid preparation and characterization." Powder technology 196, no. 2 (2009): 89-101. https://doi.org/10.1016/j.powtec.2009.07.025

[29] Choi, S. US, and Jeffrey A. Eastman. Enhancing thermal conductivity of fluids with nanoparticles. No. ANL/MSD/CP84938; CONF-951135-29. Argonne National Lab., IL (United States), 1995.

[30] Choi, S. U. S., D. A. Singer, and H. P. Wang. "Developments and applications of non-Newtonian flows." Asme Fed 66 (1995): 99-105.

[31] Peng, Qiang, Xiaoxi Yang, Jing Ding, Xiaolan Wei, and Jianping Yang. "Design of new molten salt thermal energy storage material for solar thermal power plant." Applied Energy 112 (2013): 682-689. https://doi.org/10.1016/i.apenergy.2012.10.048

[32] Silverman, M. D., W. R. Huntley, and H. E. Robertson. Heat transfer measurements in a forced convection loop with two molten-fluoride salts: LiF--BeF 2--ThF 2--UF 4 and eutectic NaBF 4--NaF. No. ORNL/TM--5335. Oak Ridge National Lab., 1976. https://doi.org/10.2172/7143311

[33] Cooke, J. W., and B. Cox. Forced-convection heat-transfer measurements with a molten fluoride salt mixture flowing in a smooth tube. No. ORNL-TM-4079. Oak Ridge National Lab., Tenn.(USA), 1973. https://doi.org/10.2172/4486196

[34] Hoffman, H. W., and J. Lones. Fused Salt Heat Transfer. Part II. Forced convection heat transfer in circular tubes containing NaF-KF-LiF eutectic. No. ORNL-1777. Oak Ridge National Lab., Tenn., 1955. https://doi.org/10.2172/4016896

[35] Grele, Milton D., and Louis Gedeon. Forced-convection heat-transfer characteristics of molten FLiNaK flowing in an Inconel X system. National Advisory Committee for Aeronautics, 1954. 
[36] Vriesema, B. "Aspects of molten fluorides as heat transfer agents for power generation, WTHD Report, no. 112." Delft University of Technology, The Netherlands (1979).

[37] ElShafei, Ahmed Ibrahim, Amr Guaily, and Mohammed A. Boraey. "Comparative Study of Nusselt Number Correlations for Hitec Molten Salt." In 2020 2nd Novel Intelligent and Leading Emerging Sciences Conference (NILES), pp. 282-287. IEEE, 2020. https://doi.org/10.1109/NILES50944.2020.9257940

[38] Kirst, W. E., W. M. Nagle, and J. B. Castner. "A new heat transfer medium for high temperatures." Transactions of the American institute of chemical engineers 36 (1940): 371-394.

[39] Ferng, Y. M., Kun-Yueh Lin, and Chen-Wei Chi. "CFD investigating thermal-hydraulic characteristics of FLiNaK salt as a heat exchange fluid." Applied Thermal Engineering $37 \quad$ (2012): $235-240$. https://doi.org/10.1016/i.applthermaleng.2011.11.021

[40] Cheng, Z. D., Y. L. He, and F. Q. Cui. "Numerical study of heat transfer enhancement by unilateral longitudinal vortex generators inside parabolic trough solar receivers." International journal of heat and mass transfer 55, no. 21-22 (2012): 5631-5641. https://doi.org/10.1016/i.ijheatmasstransfer.2012.05.057

[41] Chang, Chun, Adriano Sciacovelli, Zhiyong Wu, Xin Li, Yongliang Li, Mingzhi Zhao, Jie Deng, Zhifeng Wang, and Yulong Ding. "Enhanced heat transfer in a parabolic trough solar receiver by inserting rods and using molten salt as heat transfer fluid." Applied Energy 220 (2018): 337-350. https://doi.org/10.1016/i.apenergy.2018.03.091

[42] Serrano-López, Roberto, Jordi Fradera, and Santiago Cuesta-López. "Molten salts database for energy applications." Chemical Engineering and Processing: Process Intensification 73 (2013): 87-102. https://doi.org/10.1016/i.cep.2013.07.008

[43] Aslfattahi, Navid, R. Saidur, Nor Azwadi Che Sidik, Mohd Faizul Mohd Sabri, and Md Hasan Zahir. "Experimental assessment of a novel eutectic binary molten salt-based hexagonal boron nitride nanocomposite as a promising PCM with enhanced specific heat capacity." Journal of Advanced Research in Fluid Mechanics and Thermal Sciences 68, no. 1 (2020): 73-85. https://doi.org/10.37934/arfmts.68.1.7385

[44] ElShafei, Ahmed Ibrahim, Omar Khaled Sallam, Mohammed A. Boraey, and Amr Guaily. "Prediction of the Hitec Molten Salt Convective Heat Transfer Performance Using Artificial Neural Networks." In Joint European-US Workshop on Applications of Invariance in Computer Vision, pp. 646-654. Springer, Cham, 2020. https://doi.org/10.1007/978-3-030-44289-7 60

[45] Qiu, Yu, Ming-Jia Li, Meng-Jie Li, Hong-Hu Zhang, and Bo Ning. "Numerical and experimental study on heat transfer and flow features of representative molten salts for energy applications in turbulent tube flow." International Journal of Heat and Mass Transfer 135 (2019): 732-745. https://doi.org/10.1016/i.ijheatmasstransfer.2019.02.004

[46] Ansys, Inc. "ANSYS FLUENT theory guide." Canonsburg, Pa 794 (2011).

[47] Chen, Yushuang, Zhongfeng Tang, and Naxiu Wang. "Numerical prediction of turbulent convective heat transfer with molten salt in circular pipe." NURETH-16, Chicago, IL, August 30-September 4 (2015): 2015.

[48] Shih, Tsan-Hsing, William W. Liou, Aamir Shabbir, Zhigang Yang, and Jiang Zhu. "A new k- $\epsilon$ eddy viscosity model for high reynolds number turbulent flows." Computers \& fluids 24, no. $3 \quad$ (1995): $227-238$. https://doi.org/10.1016/0045-7930(94)00032-T

[49] Rezaeiha, Abdolrahim, Hamid Montazeri, and Bert Blocken. "On the accuracy of turbulence models for CFD simulations of vertical axis wind turbines." Energy $180 \quad$ (2019): 838-857. https://doi.org/10.1016/j.energy.2019.05.053

[50] Fluent, A. N. S. Y. S. "Ansys fluent theory guide." ANSYS Inc., USA 15317 (2011): 724-746. 